CARPATHIAN J. MATH.

Volume 37 (2021), No. 3,

Pages 541 - 550

\title{
Convergence of Tseng-type self-adaptive algorithms for variational inequalities and fixed point problems
}

\author{
YONGHONG YAO ${ }^{1}$, NASEER SHAHZAD ${ }^{2}$ and JEN-CHIH YAO ${ }^{3}$
}

\begin{abstract}
In this paper, we present a Tseng-type self-adaptive algorithm for solving a variational inequality and a fixed point problem involving pseudomonotone and pseudocontractive operators in Hilbert spaces. A weak convergent result for such algorithm is proved under a weaker assumption than sequentially weakly continuous imposed on the pseudomonotone operator. Some corollaries are also included.
\end{abstract}

\section{INTRODUCTION}

Let $H$ be a real Hilbert space with inner product $\langle\cdot, \cdot\rangle$ and induced norm $\|\cdot\|$. Let $C$ be a nonempty closed and convex subset of $H$.

In this paper, our work is closely related to a classical variational inequality:

$$
\text { find } x^{\dagger} \in C \text { such that }\left\langle f\left(x^{\dagger}\right), x-x^{\dagger}\right\rangle \geq 0, \forall x \in C,
$$

where $f: H \rightarrow H$ is a nonlinear operator. Here, use $\operatorname{Sol}(f, C)$ to denote the solution set of (1.1). Throughout, assume that $\operatorname{Sol}(f, C)$ is nonempty.

Variational inequalities are theoretically and algorithmically applied in various fields like particular cases convex optimization problems $([3,4])$, linear and monotone complementarity problems ([2]), equilibrium problems ([28]), fixed point problems ([27]), etc. For more information, please refer to [5, 11, 20, 21, 24].

A survey of algorithms for variational inequalities can be found in [12]. If $f(x)=$ $\nabla F(x)$ for some convex function $F: C \rightarrow C$, variational inequality (1.1) is equivalent to $\min _{C} F(x)$. This fact indicates a natural extension of the projection gradient algorithm $([17,18,19,22])$ for the constrained optimization, i.e., an iterate with the form

$$
u_{n+1}=\operatorname{proj}_{C}\left[u_{n}-\tau_{n} f\left(u_{n}\right)\right]
$$

where $\tau_{n}>0$ is stepsize and $\operatorname{proj}_{C}$ means the orthogonal projection from $H$ onto $C$.

This algorithm (1.2) is convergent under quite strong assumptions, in which $f$ must be strongly monotone and Lipschitz continuous. To avoid these difficulties, Korpelevich suggested in [16] an extragradient algorithm of the form

$$
\left\{\begin{array}{l}
v_{n}=\operatorname{proj}_{C}\left[u_{n}-\tau_{n} f\left(u_{n}\right)\right], \\
u_{n+1}=\operatorname{proj}_{C}\left[u_{n}-\tau_{n} f\left(v_{n}\right)\right] .
\end{array}\right.
$$

Extragradient algorithm (1.3) affords an available method for solving a classical monotone variational inequality. Consequently, extragradient algorithm (1.3) was applied by many scholars, who implemented it in a variety of forms; see, e.g., [7, 9, 13, 14, 15, 23]. Especially, Ceng, Teboulle and Yao [6] established the weak convergence of extragradient algorithm for solving the pseudomonotone variational inequality and fixed point problem under the

Received: 03.09.2020. In revised form: 24.05.2021. Accepted: 02.06.2021

2010 Mathematics Subject Classification. 47J25, 65K10, 90C99.

Key words and phrases. Variational inequality, fixed point, pseudomonotone operators, pseudocontractive operators, Tseng-type algorithm.

Corresponding author: Naseer Shahzad; nshahzad@kau.edu.sa 
additional hypothesis of the sequentially weak-to-strong continuity of $f$. However, this additional hypothesis is not satisfied even for the identity operator. Recently, Vuong [26] weaken this hypothesis to the sequentially weak-to-weak continuity of $f$.

At the same time, an inevitable drawback of extragradient algorithm is the need to calculate two projections onto the closed convex set $C$ in each iteration. For solving this flaw, as a transformation of extragradient algorithm (1.3) is the following remarkable procedure introduced by Tseng [25]

$$
\left\{\begin{array}{l}
v_{n}=\operatorname{proj}_{C}\left[u_{n}-\tau_{n} f\left(u_{n}\right)\right], \\
u_{n+1}=v_{n}+\tau_{n}\left[f\left(u_{n}\right)-f\left(v_{n}\right)\right] .
\end{array}\right.
$$

Here, a natural problem arises: could we extend Tseng's algorithm for solving some common problems related to variational inequalities under some weaker conditions imposed on $f$ ?

It is our main purpose in this paper that we further investigate iterative algorithm for solving pseudomonotone variational inequality and fixed point problem of pseudocontractive operators under the weaker assumption imposed on $f$. Our method bases on Tseng's algorithm and self-adaptive technique which is independent of the Lipschitz constant of $f$. We prove that the proposed algorithm weakly converges to a common solution of the pseudomonotone variational inequality and of the fixed point problem for the pseudocontractive operator $g$.

\section{PRELIMINARIES}

Let $C$ be a nonempty closed convex subset of a real Hilbert space $H$. Let $\left\{u_{n}\right\}$ be a sequence in $H . u_{n} \rightarrow z^{\dagger}$ denotes the weak convergence of $u_{n}$ to $z^{\dagger}$. $\omega_{w}\left(u_{n}\right)$ denotes the set of all weak cluster points of $\left\{u_{n}\right\}$, i.e., $\omega_{w}\left(u_{n}\right)=\left\{u^{\dagger}: \exists\left\{u_{n_{i}}\right\} \subset\left\{u_{n}\right\}\right.$ such that $u_{n_{i}} \rightarrow$ $\left.u^{\dagger}(i \rightarrow \infty)\right\}$. Recall that an operator $f: H \rightarrow H$ is said to be

- monotone if

$$
\left\langle f(x)-f\left(x^{\dagger}\right), x-x^{\dagger}\right\rangle \geq 0, \forall x, x^{\dagger} \in H .
$$

- strongly monotone if there exists some constant $\gamma>0$ such that

$$
\left\langle f(x)-f\left(x^{\dagger}\right), x-x^{\dagger}\right\rangle \geq \gamma\left\|x-x^{\dagger}\right\|^{2}, \forall x, x^{\dagger} \in H .
$$

- pseudomonotone if

$$
\left\langle f\left(x^{\dagger}\right), x-x^{\dagger}\right\rangle \geq 0 \text { implies that }\left\langle f(x), x-x^{\dagger}\right\rangle \geq 0, \forall x, x^{\dagger} \in H ;
$$

- $L$-Lipschitz continuous if there exists some constant $L>0$ such that

$$
\left\|f(x)-f\left(x^{\dagger}\right)\right\| \leq L\left\|x-x^{\dagger}\right\|, \text { for all } x, x^{\dagger} \in H .
$$

- sequently weakly continuous if $x_{n} \rightarrow \tilde{x}$ implies that $f\left(x_{n}\right) \rightarrow f(\tilde{x})$.

Recall that an operator $g: C \rightarrow C$ is said to be pseudocontractive if

$$
\left\|g(x)-g\left(x^{\dagger}\right)\right\|^{2} \leq\left\|x-x^{\dagger}\right\|^{2}+\left\|(I-g) x-(I-g) x^{\dagger}\right\|^{2}
$$

for all $x, x^{\dagger} \in C$.

Here, we use Fix $(g)$ to denote the fixed points set of $g$.

For fixed $x \in H$, there exists a unique $x^{\dagger} \in C$ satisfying $\left\|x-x^{\dagger}\right\|=\inf \{\|x-\tilde{x}\|: \tilde{x} \in C\}$. Denote $x^{\dagger}$ by $\operatorname{proj}_{C}[x]$. The projection $\operatorname{proj}_{C}$ has the following basic property: for given $x \in H$,

$$
\left\langle x-\operatorname{proj}_{C}[x], y-\operatorname{proj}_{C}[x]\right\rangle \leq 0, \forall y \in C .
$$

Applying this characteristic inequality, we have the following equivalence relation

$$
x^{\dagger} \in \operatorname{Sol}(f, C) \Leftrightarrow x^{\dagger}=\operatorname{proj}_{C}\left[x^{\dagger}-\tau f\left(x^{\dagger}\right)\right], \forall \tau>0 .
$$


In a Hilbert space $H$, we have

$$
\left\|\alpha u+(1-\alpha) u^{\dagger}\right\|^{2}=\alpha\|u\|^{2}+(1-\alpha)\left\|u^{\dagger}\right\|^{2}-\alpha(1-\alpha)\left\|u-u^{\dagger}\right\|^{2},
$$

$\forall u, u^{\dagger} \in H$ and $\forall \alpha \in[0,1]$.

Lemma 2.1 ([28]). Let $C$ be a nonempty, convex and closed subset of a Hilbert space H. Assume that $g: C \rightarrow C$ is an L-Lipschitz pseudocontractive operator. Then, for all $\tilde{u} \in C$ and $u^{\dagger} \in$ Fix $(g)$, we have

$$
\left\|u^{\dagger}-g[(1-\mu) \tilde{u}+\mu g(\tilde{u})]\right\|^{2} \leq\left\|\tilde{u}-u^{\dagger}\right\|^{2}+(1-\mu)\|\tilde{u}-g[(1-\mu) \tilde{u}+\mu g(\tilde{u})]\|^{2},
$$

where $0<\mu<\frac{1}{\sqrt{1+L^{2}}+1}$.

Lemma 2.2 ([27]). Let $C$ be a nonempty, convex and closed subset of a Hilbert space $H$. Let $g: C \rightarrow C$ be a continuous pseudocontractive operator. Then,

(i) Fix $(g) \subset C$ is closed and convex;

(ii) $g$ is demi-closedness, i.e., $u_{n} \rightarrow \tilde{z}$ and $g\left(u_{n}\right) \rightarrow z^{\dagger}$ imply that $g(\tilde{z})=z^{\dagger}$.

Lemma 2.3 ([8]). Let $C$ be a nonempty closed convex subset of a real Hilbert space $H$. Let $f: H \rightarrow H$ be a continuous and pseudomonotone operator. Then $x^{\dagger} \in S o l(f, C)$ iff $x^{\dagger}$ solves the following dual variational inequality

$$
\left\langle f\left(u^{\dagger}\right), u^{\dagger}-x^{\dagger}\right\rangle \geq 0, \forall u^{\dagger} \in C .
$$

Lemma 2.4 ([1]). Let $C$ be a nonempty closed convex subset of a real Hilbert space $H$. Let $\left\{x_{n}\right\} \subset H$ be a sequence. If the following assumptions are satisfied

(i) $\forall \tilde{x} \in C, \lim _{n \rightarrow \infty}\left\|x_{n}-\tilde{x}\right\|$ exists;

(ii) $\omega_{w}\left(x_{n}\right) \subset C$,

then $x_{n} \rightarrow u \in C$.

\section{MAIN RESULTS}

In this section, we first propose a Tseng-type algorithm for solving pseudomonotone variational inequality (1.1) and the fixed point problem for the pseudocontractive operator $g$ by using a self-adaptive stepsize search. Let $C$ be a nonempty closed convex subset of a real Hilbert space $H$. Let $f, g: H \rightarrow H$ be two nonlinear operators. Let $\left\{\gamma_{n}\right\}$ and $\left\{\mu_{n}\right\}$ be two sequences in $(0,1)$. Let $\alpha \in(0,1]$ and $\delta \in(0,1)$ be two constants.

Algorithm 3.1. Initialization: Take $u_{0} \in C$ and $\tau_{0}>0$. Set $n=0$.

Step 1. (Fixed point step) For known $u_{n}$, compute

$$
v_{n}=\left(1-\gamma_{n}\right) u_{n}+\gamma_{n} g\left[\left(1-\mu_{n}\right) u_{n}+\mu_{n} g\left(u_{n}\right)\right] .
$$

Step 2. (Tseng-type step) For known $\tau_{n}$, compute

$$
w_{n}=\operatorname{proj}_{C}\left[v_{n}-\tau_{n} f\left(v_{n}\right)\right] \text {, }
$$

and

$$
u_{n+1}=(1-\alpha) v_{n}+\alpha w_{n}+\alpha \tau_{n}\left[f\left(v_{n}\right)-f\left(w_{n}\right)\right] .
$$

Step 3. (self-adaptive step) Compute

$$
\tau_{n+1}= \begin{cases}\min \left\{\tau_{n}, \frac{\delta\left\|w_{n}-v_{n}\right\|}{\left\|f\left(w_{n}\right)-f\left(v_{n}\right)\right\|}\right\}, & \text { if } f\left(w_{n}\right) \neq f\left(v_{n}\right), \\ \tau_{n}, & \text { if } f\left(w_{n}\right)=f\left(v_{n}\right) .\end{cases}
$$

Step 4 . Set $n:=n+1$ and return to step 1 .

Remark 3.1. If at some step $w_{n}=v_{n}=\operatorname{proj}_{C}\left[v_{n}-\tau_{n} f\left(v_{n}\right)\right]$, by the equivalence relation (2.6), we deduce that $v_{n} \in \operatorname{Sol}(f, C)$. 
Remark 3.2. If choose $\alpha=1$ in (3.10), then Step 2 can be rewritten as

$$
\left\{\begin{array}{l}
w_{n}=\operatorname{proj}_{C}\left[v_{n}-\tau_{n} f\left(v_{n}\right)\right], \\
u_{n+1}=w_{n}+\tau_{n}\left[f\left(v_{n}\right)-f\left(w_{n}\right)\right],
\end{array}\right.
$$

which is exactly Tseng's method.

Remark 3.3 ([3]). By (3.11), we know that $\tau_{n}$ is monotonically decreasing. Moreover, by the $\kappa$-Lipschitz continuity of $f$, we deduce that $\frac{\delta\left\|w_{n}-v_{n}\right\|}{\left\|f\left(w_{n}\right)-f\left(v_{n}\right)\right\|} \geq \frac{\delta}{\kappa}$, which together with (3.11) implies that $\tau_{n} \geq \min \left\{\tau_{0}, \frac{\delta}{\kappa}\right\}$. Thus, the $\operatorname{limit}_{n \rightarrow \infty} \tau_{n}$ exists, denoted by $\tau^{\dagger}$. It is obviously that $\tau^{\dagger}>0$ which ensures $\tau_{n}$ strictly greater than zero at each iterative step.

Remark 3.4. If $f\left(w_{n}\right)=f\left(v_{n}\right)$, then the next iterate $u_{n+1}$ is independent of the stepsize $\tau_{n}$. In this case, we can choose $\tau_{n+1}$ to be any number between $\tau^{\dagger}$ and $\tau_{n}$.

In the sequel, we assume that the operator $f$ satisfies the following property (F): For given a sequence $\left\{u_{n}\right\} \subset H$, if $u_{n} \rightarrow u \in H$ and $\liminf _{n \rightarrow \infty}\left\|f\left(u_{n}\right)\right\|=0$, then $f(u)=0$.

Remark 3.5. It is obviously that if $f$ is sequentially weakly continuous, then $f$ satisfies the above property $(\mathrm{F})$.

Next, we prove the convergence of Algorithm 3.1.

Theorem 3.1. Assume that $f$ is a pseudomonotone and $\kappa$-Lipschitz continuous operator satisfying property $(F)$. Assume that $g$ is a pseudocontractive and L-Lipschitz continuous operator. Suppose that $\Gamma:=\operatorname{Sol}(f, C) \cap \operatorname{Fix}(g) \neq \emptyset$ and $0<\underline{\gamma}<\gamma_{n}<\bar{\gamma}<\mu_{n}<\bar{\mu}<\frac{1}{\sqrt{1+L^{2}}+1}(\forall n \geq 0)$. Then the sequence $\left\{u_{n}\right\}$ generated by Algorithm (3.10) converges weakly to some point in $\Gamma$.

Proof. Let $p \in \Gamma$. By the property (2.5) of $\operatorname{proj}_{C}$ and (3.9), we have

$$
\left\langle w_{n}-v_{n}+\tau_{n} f\left(v_{n}\right), w_{n}-p\right\rangle \leq 0 .
$$

Since $p \in \operatorname{Sol}(C, f),\left\langle f(p), w_{n}-p\right\rangle \geq 0$. This together with the pseudomonotonicity of $f$ implies that

$$
\left\langle f\left(w_{n}\right), w_{n}-p\right\rangle \geq 0 .
$$

Combining (3.12) and (3.13), we obtain

$$
\left\langle w_{n}-v_{n}, w_{n}-p\right\rangle+\tau_{n}\left\langle f\left(v_{n}\right)-f\left(w_{n}\right), w_{n}-p\right\rangle \leq 0 .
$$

It follows that

$$
\frac{1}{2}\left(\left\|w_{n}-v_{n}\right\|^{2}+\left\|w_{n}-p\right\|^{2}-\left\|v_{n}-p\right\|^{2}\right)+\tau_{n}\left\langle f\left(v_{n}\right)-f\left(w_{n}\right), w_{n}-p\right\rangle \leq 0,
$$

which yields that

$$
\left\|w_{n}-p\right\|^{2} \leq\left\|v_{n}-p\right\|^{2}-2 \tau_{n}\left\langle f\left(v_{n}\right)-f\left(w_{n}\right), w_{n}-p\right\rangle-\left\|w_{n}-v_{n}\right\|^{2} .
$$

By (3.10), we have

$$
\begin{aligned}
\left\|u_{n+1}-p\right\|^{2}= & \left\|(1-\alpha)\left(v_{n}-p\right)+\alpha\left(w_{n}-p\right)+\alpha \tau_{n}\left[f\left(v_{n}\right)-f\left(w_{n}\right)\right]\right\|^{2} \\
= & \left\|(1-\alpha)\left(v_{n}-p\right)+\alpha\left(w_{n}-p\right)\right\|^{2}+\alpha^{2} \tau_{n}^{2}\left\|f\left(v_{n}\right)-f\left(w_{n}\right)\right\|^{2} \\
& +2 \alpha(1-\alpha) \tau_{n}\left\langle v_{n}-p, f\left(v_{n}\right)-f\left(w_{n}\right)\right\rangle \\
& +2 \alpha^{2} \tau_{n}\left\langle w_{n}-p, f\left(v_{n}\right)-f\left(w_{n}\right)\right\rangle .
\end{aligned}
$$


From (2.7) and (3.15), we derive

$$
\begin{aligned}
\left\|u_{n+1}-p\right\|^{2}= & (1-\alpha)\left\|v_{n}-p\right\|+\alpha\left\|w_{n}-p\right\|^{2}-\alpha(1-\alpha)\left\|v_{n}-w_{n}\right\|^{2} \\
& +\alpha^{2} \tau_{n}^{2}\left\|f\left(v_{n}\right)-f\left(w_{n}\right)\right\|^{2}+2 \alpha^{2} \tau_{n}\left\langle w_{n}-p, f\left(v_{n}\right)-f\left(w_{n}\right)\right\rangle \\
& +2 \alpha(1-\alpha) \tau_{n}\left\langle v_{n}-p, f\left(v_{n}\right)-f\left(w_{n}\right)\right\rangle .
\end{aligned}
$$

According to (3.14) and (3.16), we obtain

$$
\begin{aligned}
\left\|u_{n+1}-p\right\|^{2} \leq & \left\|v_{n}-p\right\|-\alpha(2-\alpha)\left\|v_{n}-w_{n}\right\|^{2}+\alpha^{2} \tau_{n}^{2}\left\|f\left(v_{n}\right)-f\left(w_{n}\right)\right\|^{2} \\
& +2 \alpha(1-\alpha) \tau_{n}\left\langle v_{n}-w_{n}, f\left(v_{n}\right)-f\left(w_{n}\right)\right\rangle \\
\leq & \left\|v_{n}-p\right\|-\alpha(2-\alpha)\left\|v_{n}-w_{n}\right\|^{2}+\alpha^{2} \tau_{n}^{2}\left\|f\left(v_{n}\right)-f\left(w_{n}\right)\right\|^{2} \\
& +2 \alpha(1-\alpha) \tau_{n}\left\|v_{n}-w_{n}\right\|\left\|f\left(v_{n}\right)-f\left(w_{n}\right)\right\| .
\end{aligned}
$$

Thanks to (3.11), $\left\|f\left(w_{n}\right)-f\left(v_{n}\right)\right\| \leq \frac{\delta\left\|w_{n}-v_{n}\right\|}{\tau_{n+1}}$. It follows from (3.17) that

$$
\begin{aligned}
\left\|u_{n+1}-p\right\|^{2} \leq & \left\|v_{n}-p\right\|-\alpha(2-\alpha)\left\|v_{n}-w_{n}\right\|^{2}+\alpha^{2} \delta^{2} \frac{\tau_{n}^{2}}{\tau_{n+1}^{2}}\left\|w_{n}-v_{n}\right\|^{2} \\
& +2 \alpha(1-\alpha) \delta \frac{\tau_{n}}{\tau_{n+1}}\left\|v_{n}-w_{n}\right\|^{2} \\
= & \left\|v_{n}-p\right\|-\alpha\left[2-\alpha-\alpha \delta^{2} \frac{\tau_{n}^{2}}{\tau_{n+1}^{2}}-2(1-\alpha) \delta \frac{\tau_{n}}{\tau_{n+1}}\right]\left\|v_{n}-w_{n}\right\|^{2}
\end{aligned}
$$

By Remark 3.3, we deduce

$$
\lim _{n \rightarrow \infty}\left[2-\alpha-\alpha \delta^{2} \frac{\tau_{n}^{2}}{\tau_{n+1}^{2}}-2(1-\alpha) \delta \frac{\tau_{n}}{\tau_{n+1}}\right]=2-\alpha-\alpha \delta^{2}-2(1-\alpha) \delta>0 .
$$

So, there exists $\theta>0$ and $N$ such that

$$
2-\alpha-\alpha \delta^{2} \frac{\tau_{n}^{2}}{\tau_{n+1}^{2}}-2(1-\alpha) \delta \frac{\tau_{n}}{\tau_{n+1}} \geq \theta
$$

when $n \geq N$.

In combination with (3.18), we get

$$
\left\|u_{n+1}-p\right\|^{2} \leq\left\|v_{n}-p\right\|-\alpha \theta\left\|v_{n}-w_{n}\right\|^{2} .
$$

Set $t_{n}=\left(1-\mu_{n}\right) u_{n}+\mu_{n} g\left(u_{n}\right)$ for all $n \geq 0$. By (3.8) and (2.7), we obtain

$$
\begin{aligned}
\left\|v_{n}-p\right\|^{2}= & \left\|\left(1-\gamma_{n}\right)\left(u_{n}-p\right)+\gamma_{n}\left[g\left(t_{n}\right)-p\right]\right\|^{2} \\
= & \left(1-\gamma_{n}\right)\left\|u_{n}-p\right\|^{2}+\gamma_{n}\left\|g\left(t_{n}\right)-p\right\|^{2} \\
& -\gamma_{n}\left(1-\gamma_{n}\right)\left\|u_{n}-g\left(t_{n}\right)\right\|^{2} .
\end{aligned}
$$

Applying Lemma 2.1, we derive

$$
\begin{aligned}
\left\|g\left(t_{n}\right)-p\right\|^{2} & =\left\|g\left[\left(1-\mu_{n}\right) u_{n}+\mu_{n} g\left(u_{n}\right)\right]-p\right\|^{2} \\
& \leq\left\|u_{n}-p\right\|^{2}+\left(1-\mu_{n}\right)\left\|u_{n}-g\left(t_{n}\right)\right\|^{2} .
\end{aligned}
$$

Combining (3.20) and (3.21), we obtain

$$
\left\|v_{n}-p\right\|^{2} \leq\left\|u_{n}-p\right\|^{2}+\left(\gamma_{n}-\mu_{n}\right) \gamma_{n}\left\|u_{n}-g\left(t_{n}\right)\right\|^{2},
$$

which results, together with (3.19), that

$$
\left\|u_{n+1}-p\right\|^{2} \leq\left\|u_{n}-p\right\|^{2}-\left(\mu_{n}-\gamma_{n}\right) \gamma_{n}\left\|u_{n}-g\left(t_{n}\right)\right\|^{2}-\alpha \theta\left\|v_{n}-w_{n}\right\|^{2},
$$

which can be transformed into

$$
\left(\mu_{n}-\gamma_{n}\right) \gamma_{n}\left\|u_{n}-g\left(t_{n}\right)\right\|^{2}+\alpha \theta\left\|v_{n}-w_{n}\right\|^{2} \leq\left\|u_{n}-p\right\|^{2}-\left\|u_{n+1}-p\right\|^{2} .
$$


From inequalities (3.23) and (3.24), we can conclude the following conclusions:

(r1): The sequence $\left\{\| u_{n}-p\right\}$ is monotonically decreasing and hence $\lim _{n \rightarrow \infty}\left\|u_{n}-p\right\|$ exists. Thus, the sequence $\left\{u_{n}\right\}$ is bounded.

(r2): $\lim _{n \rightarrow \infty}\left\|u_{n}-g\left(t_{n}\right)\right\|=0$ and so $\lim _{n \rightarrow \infty}\left\|v_{n}-u_{n}\right\|=\lim _{n \rightarrow \infty} \gamma_{n}\left\|u_{n}-g\left(t_{n}\right)\right\|=0$.

(r3): $\lim _{n \rightarrow \infty}\left\|v_{n}-w_{n}\right\|=0$ and thus $\lim _{n \rightarrow \infty}\left\|f\left(v_{n}\right)-f\left(w_{n}\right)\right\|=0$ due to the Lipschitz continuity of $f$.

By the boundedness of the sequence $\left\{u_{n}\right\}$, we obtain the following results:

(r4): the sequence $\left\{v_{n}\right\}$ is bounded by (3.22) and $\gamma_{n}<\mu_{n}$.

(r5): the sequence $\left\{w_{n}\right\}$ is bounded because of $\left\|w_{n}\right\| \leq\left\|v_{n}\right\|+\tau_{n}\left\|f\left(v_{n}\right)\right\|$ by (3.9).

Since $f$ is $\kappa$-Lipschitz continuous, we have

$$
\begin{aligned}
\left\|u_{n}-g\left(u_{n}\right)\right\| & \leq\left\|u_{n}-g\left(t_{n}\right)\right\|+\left\|g\left(t_{n}\right)-g\left(u_{n}\right)\right\| \\
& \leq\left\|u_{n}-g\left(t_{n}\right)\right\|+\kappa \mu_{n}\left\|u_{n}-g\left(u_{n}\right)\right\| .
\end{aligned}
$$

It follows that

$$
\left\|u_{n}-g\left(u_{n}\right)\right\| \leq \frac{1}{1-\kappa \mu_{n}}\left\|u_{n}-g\left(t_{n}\right)\right\| \rightarrow 0
$$

and thus,

$$
\lim _{n \rightarrow \infty}\left\|u_{n}-g\left(u_{n}\right)\right\|=0 .
$$

By virtue of (3.10) and (r3), we have

$$
\lim _{n \rightarrow \infty}\left\|u_{n+1}-v_{n}\right\|=0 .
$$

Next, we show that $\omega_{w}\left(u_{n}\right) \subset \Gamma$. Pick up any $p^{\dagger} \in \omega_{w}\left(u_{n}\right)$. Then, there exists a subsequence $\left\{u_{n_{i}}\right\}$ of $\left\{u_{n}\right\}$ such that $u_{n_{i}} \rightarrow p^{\dagger}$ as $i \rightarrow \infty$. Consequently, $v_{n_{i}} \rightarrow p^{\dagger}$ and $w_{n_{i}} \rightarrow p^{\dagger}$ based on (r2) and (r3), respectively.

On account of (3.25) and Lemma 2.2, we acquire that $p^{\dagger} \in$ Fix $(g)$. Now, we only need to prove that $p^{\dagger} \in S o l(f, C)$. In view of (2.5) and $w_{n_{i}}=\operatorname{proj}_{C}\left[v_{n_{i}}-\tau_{n_{i}} f\left(v_{n_{i}}\right)\right]$, we achieve

$$
\left\langle w_{n_{i}}-v_{n_{i}}+\tau_{n_{i}} f\left(v_{n_{i}}\right), w_{n_{i}}-u\right\rangle \leq 0, \forall u \in C .
$$

It follows that

$$
\frac{1}{\tau_{n_{i}}}\left\langle v_{n_{i}}-w_{n_{i}}, u-w_{n_{i}}\right\rangle+\left\langle f\left(v_{n_{i}}\right), w_{n_{i}}-v_{n_{i}}\right\rangle \leq\left\langle f\left(v_{n_{i}}\right), u-v_{n_{i}}\right\rangle, \forall u \in C .
$$

Noting that from (r3), we have $\lim _{i \rightarrow \infty}\left\|v_{n_{i}}-w_{n_{i}}\right\|=0$. Then, by (3.27), we deduce

$$
\liminf _{i \rightarrow \infty}\left\langle f\left(v_{n_{i}}\right), u-v_{n_{i}}\right\rangle \geq 0 .
$$

Next, we consider two possible cases.

Case 1 . $\liminf \operatorname{in}_{i \rightarrow \infty}\left\|f\left(v_{n_{i}}\right)\right\|=0$. By $v_{n_{i}} \rightarrow p^{\dagger}$ and $f$ satisfying property (F), we deduce that $f\left(p^{\dagger}\right)=0$. Consequently, $p^{\dagger} \in \operatorname{Sol}(f, C)$.

Case 2. $\liminf _{i \rightarrow \infty}\left\|f\left(v_{n_{i}}\right)\right\|>0$. In terms of (3.28), we obtain

$$
\liminf _{i \rightarrow \infty}\left\langle\left(f\left(v_{n_{i}}\right)\right)^{0}, u-v_{n_{i}}\right\rangle \geq 0
$$

where $\left(f\left(v_{n_{i}}\right)\right)^{0}$ means the unit vector of $f\left(v_{n_{i}}\right)$, that is, $\left(f\left(v_{n_{i}}\right)\right)^{0}=\frac{f\left(v_{n_{i}}\right)}{\left\|f\left(v_{n_{i}}\right)\right\|}$ (note that for each $i \geq 0, f\left(v_{n_{i}}\right) \neq 0$, otherwise, $v_{n_{i}} \in \operatorname{Sol}(f, C)$ and $\left.p^{\dagger} \in \operatorname{Sol}(f, C)\right)$.

Thanks to (3.29), we can choose a positive real numbers sequence $\left\{\epsilon_{i}\right\}$ satisfying $\epsilon_{i} \rightarrow 0$ as $i \rightarrow \infty$. For each $\epsilon_{i}$, there exists the smallest positive integer $N_{i}$ such that

$$
\left\langle\left(f\left(v_{n_{i}}\right)\right)^{0}, u-v_{n_{i}}\right\rangle+\epsilon_{i} \geq 0, \forall i \geq N_{i} .
$$


It follows that

$$
\left\langle f\left(v_{n_{i}}\right), u-v_{n_{i}}\right\rangle+\epsilon_{i}\left\|f\left(v_{n_{i}}\right)\right\| \geq 0, \forall i \geq N_{i} .
$$

Set $\hat{v}_{n_{i}}=\frac{f\left(v_{n_{i}}\right)}{\left\|f\left(v_{n_{i}}\right)\right\|^{2}}$. Thus, we have $\left\langle f\left(v_{n_{i}}\right), \hat{v}_{n_{i}}\right\rangle=1$ for each $i$. From (3.30), we deduce

$$
\left\langle f\left(v_{n_{i}}\right), u+\epsilon_{i}\left\|f\left(v_{n_{i}}\right)\right\| \hat{v}_{n_{i}}-v_{n_{i}}\right\rangle \geq 0, \forall i \geq N_{i} .
$$

Since $f$ is pseudomonotone, it follows from (3.31) that

$$
\left\langle f\left(u+\epsilon_{i}\left\|f\left(v_{n_{i}}\right)\right\| \hat{v}_{n_{i}}\right), u+\epsilon_{i}\left\|f\left(v_{n_{i}}\right)\right\| \hat{v}_{n_{i}}-v_{n_{i}}\right\rangle \geq 0, \forall i \geq N_{i} .
$$

Note that $\lim _{i \rightarrow \infty}\left\|\epsilon_{i}\right\| f\left(v_{n_{i}}\right)\left\|\hat{v}_{n_{i}}\right\|=\lim _{i \rightarrow \infty} \epsilon_{i}=0$. Thus, taking the limit as $i \rightarrow \infty$ in (3.32), we obtain

$$
\left\langle f(u), u-p^{\dagger}\right\rangle \geq 0 \text {. }
$$

Applying Lemma 2.1 to (3.33), we conclude that $p^{\dagger} \in S o l(f, C)$.

Finally, we show that the entire sequence $\left\{u_{n}\right\}$ converges weakly to $p^{\dagger}$. As a matter of fact, we have the following facts in hand:

(i) $\forall p \in \Gamma, \lim _{n \rightarrow \infty}\left\|u_{n}-p\right\|$ exists;

(ii) $w_{\omega}\left(u_{n}\right) \subset \Gamma$;

(iii) $p^{\dagger} \in w_{\omega}\left(u_{n}\right)$.

Thus, by Lemma 2.4, we deduce that the sequence $\left\{u_{n}\right\}$ weakly converges to $p^{\dagger} \in \Gamma$. This completes the proof.

Remark 3.6. It is obviously that monotonicity implies pseudo-monotonicity. Hence, our theorem holds when the involved operator $f$ is monotone.

Based on Algorithm 3.1 and Theorem 3.1, we can obtain the following algorithms and the corresponding corollaries.

Algorithm 3.2. Initialization: Take $u_{0} \in C$ and $\tau_{0}>0$. Set $n=0$.

Step 1. For known $u_{n}$ and $\tau_{n}$, compute

$$
w_{n}=\operatorname{proj}_{C}\left[u_{n}-\tau_{n} f\left(u_{n}\right)\right],
$$

and

$$
u_{n+1}=(1-\alpha) u_{n}+\alpha w_{n}+\alpha \tau_{n}\left[f\left(u_{n}\right)-f\left(w_{n}\right)\right] .
$$

Step 2. Compute

$$
\tau_{n+1}= \begin{cases}\min \left\{\tau_{n}, \frac{\delta\left\|w_{n}-u_{n}\right\|}{\left\|f\left(w_{n}\right)-f\left(u_{n}\right)\right\|}\right\}, & \text { if } f\left(w_{n}\right) \neq f\left(u_{n}\right), \\ \tau_{n}, & \text { else. }\end{cases}
$$

Step 3. Set $n:=n+1$ and return to step 1 .

Corollary 3.1. Assume that $f$ is a pseudomonotone and $\kappa$-Lipschitz continuous operator satisfying property $(F)$. Suppose that $\operatorname{Sol}(f, C) \neq \emptyset$. Then the sequence $\left\{u_{n}\right\}$ generated by Algorithm 3.2 converges weakly to some point in $\operatorname{Sol}(f, C)$.

Algorithm 3.3. Initialization: Take $u_{0} \in C$ and $\tau_{0}>0$. Set $n=0$.

Step 1. For known $u_{n}$, compute

$$
u_{n+1}=\left(1-\gamma_{n}\right) u_{n}+\gamma_{n} g\left[\left(1-\mu_{n}\right) u_{n}+\mu_{n} g\left(u_{n}\right)\right] .
$$

Step 2. Set $n:=n+1$ and return to step 1 .

Corollary 3.2. Assume that $g$ is a pseudocontractive and L-Lipschitz continuous operator. Suppose that Fix $(g) \neq \emptyset$ and $0<\underline{\gamma}<\gamma_{n}<\bar{\gamma}<\mu_{n}<\bar{\mu}<\frac{1}{\sqrt{1+L^{2}}+1}(\forall n \geq 0)$. Then the sequence $\left\{u_{n}\right\}$ generated by Algorithm 3.3 converges weakly to some point in Fix $(g)$. 


\section{APPLICATION TO COMPUTING DYNAMIC USER EQUILIBRIA}

In this section, we apply Algorithm 3.2 to compute dynamic user equilibria ([10]).

Let $\mathcal{P}$ be set of paths in the network. $\mathcal{W}$ be set of O-D pairs in the network, $Q_{i j}$ be fxed O-D demand between $(i, j) \in \mathcal{W}, \mathcal{P}_{i j}$ be subset of paths that connect O-D pair $(i, j), t$ be continuous time parameter in a fxed time horizon $\left[t_{0}, t_{1}\right], h_{p}(t)$ be departure rate along path $p$ at time $t, h(t)$ be complete vector of departure rates $h(t)=\left(h_{p}(t): p \in \mathcal{P}\right), \Psi_{p}(t, h)$ be travel cost along path $\mathrm{p}$ with departure time $t$, under departure profile $h, v_{i j}(h)$ be minimum travel cost between O-D pair $(i, j)$ for all paths and departure times.

Assume that $h_{p}(\cdot) \in L_{+}^{2}\left[t_{0}, t_{1}\right]$ and $h(\cdot) \in\left(L_{+}^{2}\left[t_{0}, t_{1}\right]\right)^{|\mathcal{P}|}$. Define the effective delay operator $\Psi:\left(L_{+}^{2}\left[t_{0}, t_{1}\right]\right)^{|\mathcal{P}|} \rightarrow\left(L_{+}^{2}\left[t_{0}, t_{1}\right]\right)^{|\mathcal{P}|}$ as follows:

$$
h(\cdot)=\left\{h_{p}(\cdot), p \in \mathcal{P}\right\} \mapsto \Psi(h)=\left\{\Psi_{p}(\cdot, h), p \in \mathcal{P}\right\}
$$

The travel demand satisfaction constraint satisfies

$$
Q_{i j}=\sum_{p \in \mathcal{P}_{i j}} \int_{t_{0}}^{t_{1}} h_{p}(t) d t, \forall(i, j) \in \mathcal{W} .
$$

Then, the set of feasible path departure vector can be expressed as

$$
\Lambda=\left\{h \geq 0: \sum_{p \in \mathcal{P}_{i j}} \int_{t_{0}}^{t_{1}} h_{p}(t) d t, \forall(i, j) \in \mathcal{W}\right\} \subset\left(L^{2}\left[t_{0}, t_{1}\right]\right)^{|\mathcal{P}|} .
$$

Recall that a vector of departures $h^{*} \in \Lambda$ is a dynamic user equilibrium with simultaneous route and departure time choice if

$$
h_{p}^{*}(t)>0, p \in \mathcal{P}_{i j} \Rightarrow \Psi_{p}\left(t, h^{*}\right)=v_{i j}\left(h^{*}\right), \text { for almost everyt } \in\left[t_{0}, t_{1}\right] .
$$

Note that (4.34) is equivalent to the following variational inequality ([10])

$$
\left\langle\Psi\left(h^{*}\right), h-h^{*}\right\rangle \geq 0, \forall h \in \Lambda .
$$

Based on Algorithm 3.2, we have the following algorithm.

Algorithm 4.1. Initial path flow $u_{0} \in\left(L^{2}\left[t_{0}, t_{1}\right]\right)^{|\mathcal{P}|}$ and $\tau_{0}>0$. Set $n=0$.

Step 1. For known $u_{n}$ and $\tau_{n}$, compute the effective path delays $\Psi_{p}\left(t, u_{n}\right)$ and

$$
w_{n}=\operatorname{proj}_{\Lambda}\left[u_{n}-\tau_{n} \Psi\left(u_{n}\right)\right] .
$$

Step 2. Compute the effective path delays $\Psi_{p}\left(t, w_{n}\right)$ and

$$
u_{n+1}=(1-\alpha) u_{n}+\alpha w_{n}+\alpha \tau_{n}\left[\Psi\left(u_{n}\right)-\Psi\left(w_{n}\right)\right] .
$$

Step 3. Compute

$$
\tau_{n+1}= \begin{cases}\min \left\{\tau_{n}, \frac{\delta\left\|w_{n}-u_{n}\right\|}{\left\|\Psi\left(w_{n}\right)-\Psi\left(u_{n}\right)\right\|}\right\}, & \text { if } \Psi\left(w_{n}\right) \neq \Psi\left(u_{n}\right), \\ \tau_{n}, & \text { else. }\end{cases}
$$

Step 4. Set $n:=n+1$ and return to step 1 .

If the delay operator $\Psi$ is Lipschitz continuous and pseudomonotone, then we can apply Algorithm 4.1 to compute dynamic user equilibria. It should be pointed out that Algorithm 4.1 requires two evaluations of the delay operator $\Psi$. It is clear that this procedure is the most costly step in the implementation of Algorithm 4.1.

Acknowledgements. Yonghong Yao was partially supported by the grant TD13-5033. Jen-Chih Yao was partially supported by the Grant MOST 106-2923-E-039-001-MY3. 


\section{REFERENCES}

[1] Abbas, B., Attouch, H.; Svaiter, B. F. Newton-like dynamics and forward-backward methods for structured monotone inclusions in Hilbert spaces. J. Optim. Theory Appl. 161 (2014), 331-360.

[2] Bauschke, H. H.; Combettes, P. L., Convex Analysis and Monotone Operator Theory in Hilbert Spaces. Springer, Berlin 2011.

[3] Bot, R. I.; Csetnek, E. R.; Vuong, P. T. The forward-backward-forward method from continuous and discrete perspective for pseudo-monotone variational inequalities in Hilbert spaces. Eur. J. Oper. Res. 287 (2020), 49-60.

[4] Cai, X.; Gu, G.; He, B. On the $O(1 / t)$ convergence rate of the projection and contraction methods for variational inequalities with Lipschitz continuous monotone operators. Comput. Optim. Appl. 57 (2014), 339-363.

[5] Ceng, L. C.; Petruşel, A.; Qin, X.; Yao, J. C. A modified inertial subgradient extragradient method for solving pseudomonotone variational inequalities and common fixed point problems. Fixed Point Theory 21 (2020), 93-108.

[6] Ceng, L. C.; Teboulle, M.; Yao, J. C. Weak convergence of an iterative method for pseudomonotone variational inequalities and fixed-point problems. J. Optim. Theory Appl. 146 (2010), 19-31.

[7] Censor, Y.; Gibali, A.; Reich, S. Extensions of Korpelevich's extragradient method for solving the variational inequality problem in Euclidean space. Optim. 61 (2012), 1119-1132.

[8] Cottle, R. W.; Yao, J. C. Pseudomonotone complementarity problems in Hilbert space. J. Optim. Theory Appl. 75 (1992), 281-295.

[9] Dong, Q. L.; Lu, Y. Y.; Yang, J. The extragradient algorithm with inertial effects for solving the variational inequality. Optim. 65 (2016), 2217-2226.

[10] Friesz, T. L.; Han, K. The mathematical foundations of dynamic user equilibrium. Transportation Research Part B: Methodological 126 (2019), 309-328.

[11] Gibali, A.; Reich, S.; Zalas, R. Iterative methods for solving variational inequalities in Euclidean space. J. Fixed Point Theory Appl. 17 (2015), 775-811.

[12] Harker, P. T.; Pang, J. S. Finite dimensional variational inequalities and nonlinear complementarity problems: a survey of theory, algorithms and applications. Math. Programming 48 (1990), 161-220.

[13] Hieu, D. V.; Anh, P. K.; Muu, L. D. Modified extragradient-like algorithms with new stepsizes for variational inequalities. Comput. Optim. Appl. 73 (2019), 913-932

[14] Iusem, A. N.; Svaiter, B. F. A variant of Korpelevich's method for variational inequalities with a new search strategy. Optim. 42 (1997), 309-321.

[15] Khanh, P. D. A modified extragradient method for infinite-dimensional variational inequalities. Acta. Math. Vietnam. 41 (2016), 251-263.

[16] Korpelevich, G. M. The extragradient method for finding saddle points and other problems. Metody 12 (1976), 747-756.

[17] Mainge, P. E. Numerical approach to monotone variational inequalities by a one-step projected reflected gradient method with line-search procedure. Comput. Math. Appl. 3 (2016), 720-728.

[18] Mainge, P. E.; Gobinddass, M. L. Convergence of one-step projected gradient methods for variational inequalities. J. Optim. Theory Appl. 171 (2016), 146-168.

[19] Malitsky, Y. V. Projected reflected gradient method for variational inequalities. SIAM J. Optim. 25 (2015), 502-520.

[20] Shehu, Y.; Gibali, A.; Sagratella, S. Inertial projection-type methods for solving quasi-variational inequalities in real Hilbert spaces. J. Optim. Theory Appl. 184 (2020), 877-894.

[21] Shehu, Y.; Li, X. H.; Dong, Q. L. An efficient projection-type method for monotone variational inequalities in Hilbert spaces. Numer. Algor. 84 (2020), 365-388.

[22] Solodov, M. V.; Svaiter, B. F. A new projection method for variational inequality problems. SIAM J. Control Optim. 37 (1999), 765-776.

[23] Thong, D. V.; Hieu, D. V. New extragradient methods for solving variational inequality problems and fixed point problems. J. Fixed Point Theory Appl. 20 (2018), Art. ID 129.

[24] Thong, D. V.; Vinh, N. T.; Cho, Y. J. A strong convergence theorem for Tsengs extragradient method for solving variational inequality problems. Optim. Lett. 14 (2020), 1157-1175.

[25] Tseng, P. A modified forward-backward splitting method for maximal monotone mappings. SIAM J. Control Optim. 38 (2000), 431-446.

[26] Vuong, P. T. On the weak convergence of the extragradient method for solving pseudomonotone variational inequalities. J. Optim. Theory Appl. 176 (2018), 399-409.

[27] Zhou, H. Strong convergence of an explicit iterative algorithm for continuous pseudocontractions in Banach spaces. Nonlinear Anal. 70 (2009), 4039-4046.

[28] Zhu, L. J.; Yao, Y.; Postolache, M. Projection methods with linesearch technique for pseudomonotone equilibrium problems and fixed point problems. U.P.B. Sci. Bull., Series A, in press. 
${ }^{1}$ TIANGONG UNIVERSITY

SCHOOL OF MATHEMATICAL SCIENCES

TIANJIN 300387, CHINA

NORTh MinZu UnIVERSITY

THE KEY LABORATORY OF INTELLIGENT INFORMATION

AND Big Data Processing of NingXia Province

YINCHUAN 750021, CHINA

E-mail address: yyhtgu@hotmail. com

${ }^{2}$ Department of Mathematics

King ABDUlaZIZ UNIVERSITY

P. O. B. 80203, JEDDAH 21589, SAUdi ARABIA

E-mail address: nshahzad@kau.edu.sa

${ }^{3}$ China Medical UNIVERsity

CENTER FOR GENERAL EDUCATION

TAICHUNG, 40402, TAIWAN

E-mail address: yao jc@mail . cmu. edu.tw 